\title{
Physiological importance of RNA and protein mobility in the cell nucleus
}

\author{
Tom Misteli
}

Accepted: 27 October 2007 / Published online: 10 November 2007 (C) Springer-Verlag 2007

\begin{abstract}
Trafficking of proteins and RNAs is essential for cellular function and homeostasis. While it has long been appreciated that proteins and RNAs move within cells, only recently has it become possible to visualize trafficking events in vivo. Analysis of protein and RNA motion within the cell nucleus have been particularly intriguing as they have revealed an unanticipated degree of dynamics within the organelle. These methods have revealed that the intranuclear trafficking occurs largely by energy-independent mechanisms and is driven by diffusion. RNA molecules and non-DNA binding proteins undergo constrained diffusion, largely limited by the spatial constraint imposed by chromatin, and chromatin binding proteins move by a stop-andgo mechanism where their free diffusion is interrupted by random association with the chromatin fiber. The ability and mode of motion of proteins and RNAs has implications for how they find nuclear targets on chromatin and in nuclear subcompartments and how macromolecular complexes are assembled in vivo. Most importantly, the dynamic nature of proteins and RNAs is emerging as a means to control physiological cellular responses and pathways.
\end{abstract}

Keywords Nuclear architecture - Dynamics · Diffusion · RNA

T. Misteli $(\bowtie)$

National Cancer Institute, National Institutes of Health,

41 Library Drive, Bethesda, MD 20892, USA

e-mail: mistelit@mail.nih.gov

\section{Introduction}

It is an irony of science that the discovery of fundamental principles is often delayed. A case in point is the cell biological investigation of the cell nucleus. The nucleus is arguably the functionally most prominent cellular organelle as it houses the vast majority of the genetic information and is the exclusive site of essential processes including transcription, RNA processing, replication and DNA repair. Yet, our understanding of how the nucleus is organized and how nuclear architecture contributes to cellular function is only now emerging and still lags behind that of most other cellular compartments. Fortunately, pioneering studies over the past decade have uncovered several of the fundamental principles which determine nuclear architecture and function (Lanctot et al. 2007; Misteli 2001; Spector 2003). These studies have revealed the presence of distinct structural elements, particularly lamin proteins and possibly short actin filaments, within the nucleus and they have led to the identification of a large number of distinct nuclear subcompartments in which specific nuclear functions, such as the synthesis and processing of ribosomal RNA in the nucleolus, occur (Burke and Stewart 2006; Gruenbaum et al. 2005; Handwerger and Gall 2006; Hernandez-Verdun 2006; Lamond and Sleeman 2003) (Fig. 1a). In addition, it has become clear that the genome is non-randomly organized within the spatial confines of the nucleus with specific chromosomes and genes preferentially localizing to specific sites (Lanctot et al. 2007; Meaburn and Misteli 2007) (Fig. 1b, c). These observations paint a picture of a highly complex and heterogeneous cell nucleus. They also indicate that some properties of the nucleus are counterintuitive. For example, despite the requirement to accommodate $\sim 2 \mathrm{~m}$ of DNA within the cell nucleus of a diameter of typically 

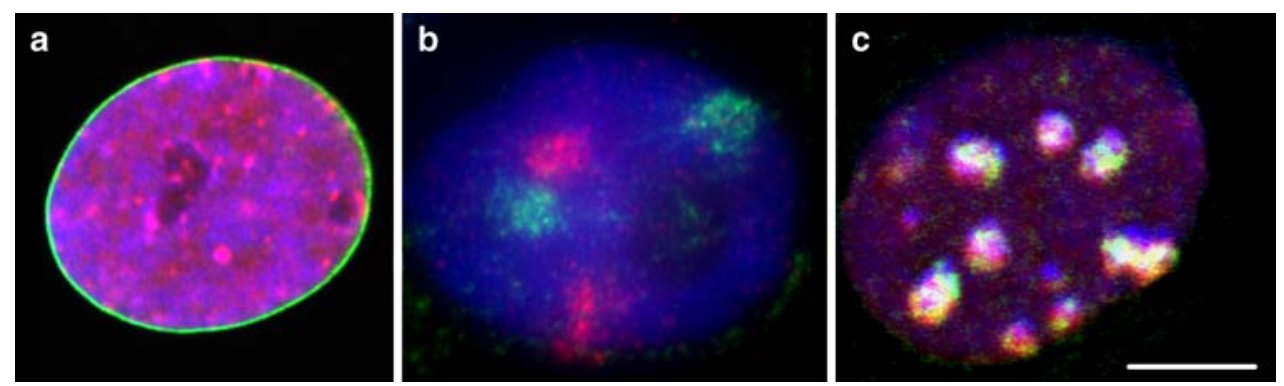

Fig. 1 Nuclear architecture and function. The mammalian cell nucleus contains a large number of non-random and structural features. a Nuclear lamins (green) form a network of architectural elements at the periphery. This nuclear lamina is implicated in mechanical support of the nucleus, organization of chromatin (red/blue) in the nuclear interior, as a platform for signaling events and in the sequestration of nuclear proteins. b, c Genomes are non-randomly organized within the

$\sim 10 \mu \mathrm{m}$, chromatin is estimated to only occupy about $15 \%$ of the nuclear volume.

A key question in understanding nuclear architecture is how protein and RNAs move within the nucleus. This is a key issue if we are to gain a full appreciation of what the nuclear environment is like. More importantly, the mode of nuclear motion of molecules has physiological implications as it affects how proteins find their target sequences in the genome, how RNAs are exported from the nucleus upon transcription and how proteins are sequestered within the nucleus for regulatory purposes. Some initial insights into these issues are summarized in broad terms here.

\section{The motion of RNA and proteins in the cell nucleus}

We have long known that proteins and RNAs must be able to move within the cell nucleus. Messenger RNAs traverse the nucleus and are promptly exported from the nucleus after their synthesis and complete processing. Newly synthesized proteins on the other hand are imported into the nucleus and rapidly disperse throughout its volume, implying effective intranuclear trafficking. Furthermore, proteins rapidly relocalize within the nucleus upon experimental or physiological changes in conditions. For example, inhibition of protein synthesis leads to rapid and dramatic relocalization of a large number of nucleolar proteins (Andersen et al. 2005), and pre-mRNA splicing factors swiftly respond to transcriptional activation and inhibition (Jiménez-García and Spector 1993; Misteli et al. 1997). How proteins and RNAs move within the nucleus and how they find their targets, however, was unknown for long. It was not clear whether movements are energy dependent, whether they occur by directed transport or what the speed of intranuclear trafficking is. These fundamental questions have recently been answered (Misteli 2001). nucleus. b Each chromosome occupies a distinct chromosome territory (red chromosome 14; green chromosome 17 in human breast epithelial cells). c Pericentromeric heterochromatin regions (white) from multiple chromosomes often cluster into chromocenters. Bar $6.3 \mu \mathrm{m}$. Image courtesy: a Gianluca Pegoraro, b Karen Meaburn, c Manjari Mazumdar, all National Cancer Institute, NIH

The investigation of intranuclear protein and RNA dynamics was made possible by the development of techniques to visualize and measure the motion of these molecules by time-lapse microscopy. Tracking of proteins by photobleaching methods revealed a surprising degree and speed of intranuclear trafficking. The analysis of tracers such as GFP or dextran which do not undergo specific interactions with DNA or other proteins indicated that the nuclear environment is permissive for rapid diffusional motion (Görisch et al. 2005; Misteli 2001; Seksek et al. 1997). The diffusion of molecules within the nucleus is only limited by the steric constraints imposed by chromatin and nuclear bodies (Görisch et al. 2005; Misteli 2001; Seksek et al. 1997). The typically measured diffusion coefficients of $10-100 \mu \mathrm{m}^{2} \mathrm{~s}^{-1}$ are similar to those observed in the cytoplasm and are only $\sim 5$ times less than that in solution. As expected for diffusion, this rapid motion is energy-independent and non-directional.

RNA motion within the nucleus is similarly rapid and non-directional. Several methodological approaches involving fluorescently labeled, microinjected, engineered or endogenous RNAs demonstrate that ribosomal RNAs as well as polyA-RNAs move freely in a non-directional manner with a diffusion coefficient of $0.03-0.1 \mu \mathrm{m}^{2} \mathrm{~s}^{-1}$ within the nucleus (Ritland-Politz et al. 2006; Shav-Tal et al. 2004). A diffusion coefficient of this magnitude is sufficient to ensure transport of an RNA particle from deep within the nucleus to the cytoplasm within a few minutes, consistent with biochemical observations on kinetics of RNA maturation and transport. Thus energy-independent, diffusionbased movement of RNA particles alone can account for the observed kinetics of RNA export, and no active mechanisms are required to ensure rapid export. An impressive demonstration of the non-directional motion of mRNA comes from studies in which a nascent RNA is visualized at its site of transcription and its export to the cytoplasm mea- 
sured in living cells (Ritland-Politz et al. 2006; Shav-Tal et al. 2004). These studies show that RNAs synthesized from genes positioned in proximity to the nuclear envelope diffuse away from their site of synthesis in all directions rather than follow a direct path to the nearest nuclear pore. This observation powerfully demonstrates the non-directed, diffusion-based motion of RNAs in the nucleus.

\section{Trafficking as a means of targeting}

One of the conceptually most challenging problems in cell biology is the question of how molecules find their specific targets within a cell or within an organelle. This problem is particularly complex in the cell nucleus where transcriptional regulators need to find their specific target genes amongst the myriad of potential binding sites within the genome. Somewhat counter-intuitively, the non-directional, but rapid, motion of proteins within the cell nucleus provides a means to ensure targeting of proteins to specific genome locations (Misteli 2001). The power of targeting by random diffusion within the nucleus is best illustrated when considering how a transcription factor finds its target genes but it also applies to targeting of proteins to nuclear compartments or any other nuclear site.

Proteins a priori do not know where their targets are and we do know of any directed transport systems, such as a molecular motor-based mechanism, that would bring a factor to a specific gene or a specific location within the nucleus. Thus, the only way for a transcription factor to find its target is to scan the genome. The intrinsic ability of proteins to rapidly move within the nucleus by diffusion-based mechanisms permits such genome scanning (Misteli 2001). This occurs by a transcription factor freely diffusing within the nucleoplasm until it interacts by chance with chromatin. The molecule will now probe whether the sequence it has encountered is a specific binding site such as in the promoter of one of its target genes. If it is, the transcription factor will be captured and stably associated with its specific target site. If the sequence is not a binding site, the molecule, after a short interaction, will dissociate from chromatin and continue its diffusional journey through the nucleus.

It might appear at first glance that such a stop-and-go model for genome scanning would be insufficiently effective. However, the observed dynamic properties of transcription factors are entirely consistent with this model. We know that the residence time of most transcription factors even on specific DNA binding sites is in the order of a few seconds and that their interaction with non-specific sites is even faster, most likely in the order of tens of milliseconds (Gorski et al. 2006; Houtsmuller et al. 1999; McNally et al. 2000; Phair et al. 2004; Sprague et al. 2006). Assuming these time scales for binding, one can calculate that it takes a single transcription factor molecule only a few minutes to search the entire genome space. Considering that most transcription factors exist in several thousand copies and have multiple target genes, their random diffusional motion is entirely sufficient to ensure a steady supply at their target genes. This assumption is further supported by direct measurement of transcription factor flux on an endogenous rRNA promoter demonstrating the collision of several hundred molecules per second (Dundr et al. 2002). Since proteins similarly move by diffusional motion within the cytoplasm, it stands to reason that the random scanning is also a key mechanism of protein targeting in the cytoplasm and represents a universal mechanism for how proteins find their targets.

A key feature, and a requirement, in a genome-scanning model of targeting is that the interactions of proteins with chromatin are transient. This has been confirmed by photobleaching methods on a large number of DNA binding proteins (Gorski et al. 2006; Phair et al. 2004; Sprague et al. 2006). The transient nature of protein-chromatin interactions is important for three reasons. First, it allows proteins to maintain a high rate of motion and thus allows faster scanning. Were protein-chromatin interactions static, they would get stuck at non-specific or incorrect binding sites which would slow down their overall motion. Second, the short-life of protein-chromatin interactions continuously makes available binding sites which can then be scanned by diffusing transcription factors. If proteins interacted for extended periods of time on chromatin, non-specific or improper binding would block access of the correct factors. Third, the dynamic dissociation allows for change. Were protein complexes permanently bound to their target sites, changes in transcriptional activity such as in response to physiological stimuli could only occur after active removal of the bound complex, presumably by dedicated and specialized molecular machinery. In contrast, in a dynamic binding model the natural flux of proteins provides a window of opportunity for association of a distinct regulator each time a bound protein or complex dissociates as part of its normal binding cycle.

\section{Protein dynamics as the key for formation of nuclear compartments}

A hallmark of the mammalian cell nucleus is the presence of distinct subnuclear compartments and domains in which particular functions occur (Handwerger and Gall 2006; Hernandez-Verdun 2006) (Fig. 2). The prototypical nuclear compartment is the nucleolus, a distinct intranuclear compartment in which ribosomal RNAs are synthesized and partially processed. Other prominent nuclear domains 
Fig. 2 Intranuclear compartments. The mammalian cell nucleus contains a larger number of distinct intranuclear compartments. The nucleolus is the site of ribosomal RNA synthesis and is a prototypical nuclear body. The complex organization of the nucleolus is revealed by multicolor staining of distinct nucleolar components. RPA43 RNA polymerase I transcription factor, Fibrillarin snRNP component, $B 23$ rRNA processing factor. Image courtesy of Miroslav Dundr, Rosalind Franklin University of Medicine and Science, Chicago

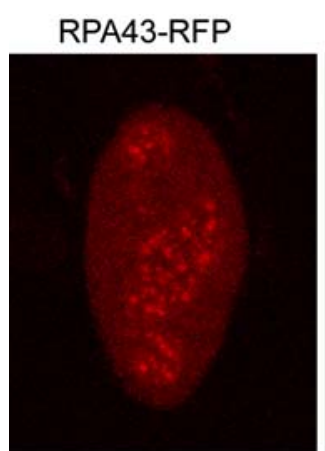

Fibrillarin-GFP
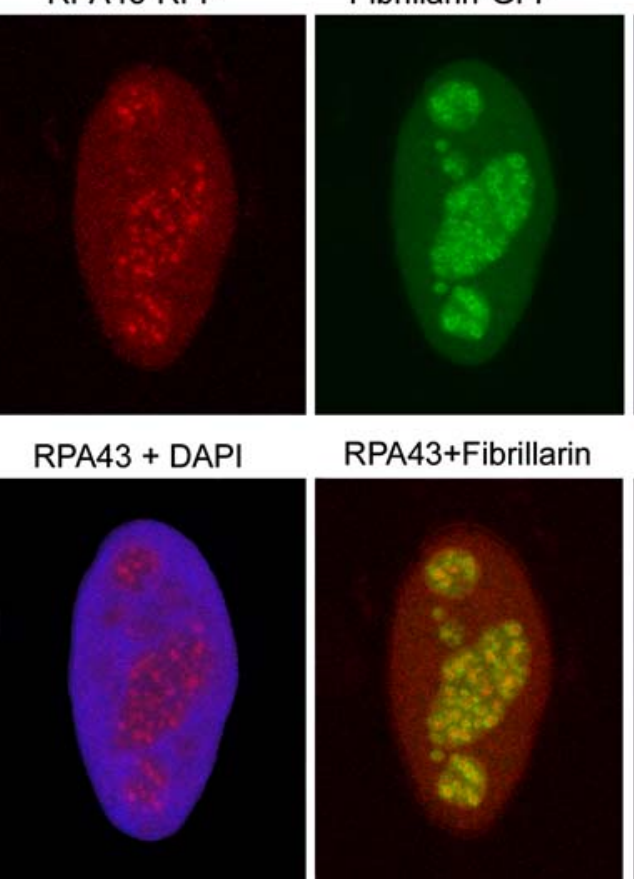

B23

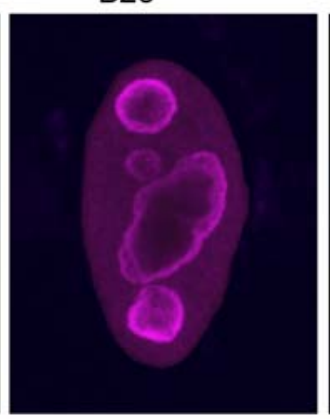

Fibrillarin + B23

DAPI

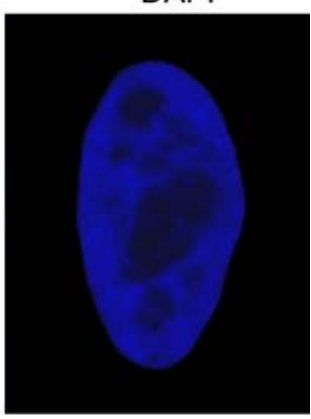

Overlay
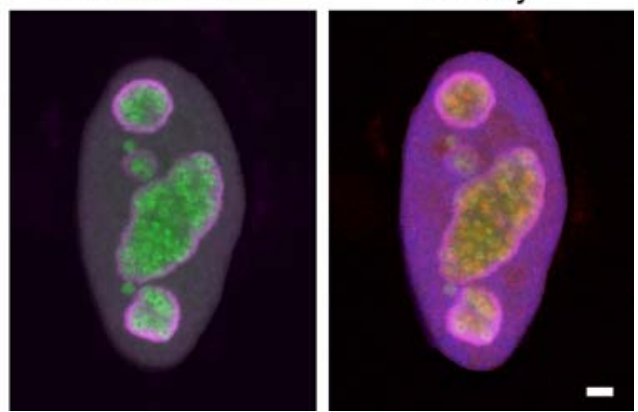

include splicing factor compartments which serve as storage and assembly sites for spliceosomal components, and the Cajal bodies which are possibly involved in maturation of small nuclear RNPs. The structure of intranuclear bodies is not determined by a membrane, and the principles underlying their biogenesis are extremely poorly understood. The recently revealed dynamic properties of proteins in nuclear compartments give a hint as to the principles involved in subnuclear compartment assembly.

The key property in understanding the formation of nuclear compartment is the remarkable fact that the association of proteins with their compartments is highly dynamic (Dundr et al. 2004; Kruhlak et al. 2000; Phair and Misteli 2000). Typical residence times of proteins within a compartment are in the seconds range. In practical terms the dynamic behavior of nuclear body proteins means that they undergo repeated rapid cycles of association and dissociation between the nuclear body and the nucleoplasm. As a consequence, a nuclear body is in perpetual flux and its structure is determined by the ratio of on-rate versus offrate of its proteins.

Given these observations, the simplest scenario to explain the formation of nuclear subdomains is a self-organization model in which the collective sum of all interactions amongst proteins in a body establishes and determines its structure (Carrero et al. 2006; Misteli 2007). In this model, nuclear bodies are essentially dynamic protein aggregates which form as a consequence of multiple, transient interactions amongst a large number of proteins. This is consistent with the lack of defining intranuclear membranes and with the absence of any known dedicated structural elements within intranuclear bodies. While dynamic observations on nucleoli, splicing factor compartments and Cajal bodies support this view, this model has not been rigorously tested experimentally. A key prediction, and a way to experimentally address this fundamental question, is that it should be possible to create nuclear bodies de novo and that any nuclear body component should be able to nucleate the formation of a body. Experimental systems to test these predictions are now available, and we are eagerly awaiting the results of these key experiments.

\section{The importance of protein and RNA dynamics in regulatory events}

The observation of dynamic properties of proteins in the cell nucleus of living cells has suggested that dynamic trafficking is an intrinsic property of proteins and RNAs. The dynamic behavior of proteins and RNAs clearly contributes to their proper function. However, is dynamic trafficking important for physiological regulation? Several observations demonstrate that dynamic trafficking of both proteins and RNA indeed can have regulatory function by several means.

Of particular importance as a regulatory mechanism is intranuclear sequestration (Fig. 3). Accumulation of a protein at a particular nuclear site or within a nuclear compartment can serve to either increase the local concentration of a factor at that site or to reduce its abundance in the rest of 


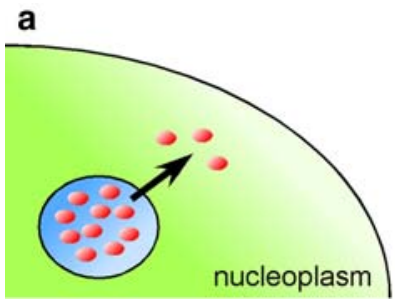

Fig. 3 Intranuclear retention and sequestration as a regulatory means. a Retention of proteins within intranuclear subcompartments regulates their nuclear concentration and their rate of nuclear export. b Sequestration of proteins at the nuclear periphery, particularly via interaction with the lamina (blue) controls their nucleoplasmic concentration and
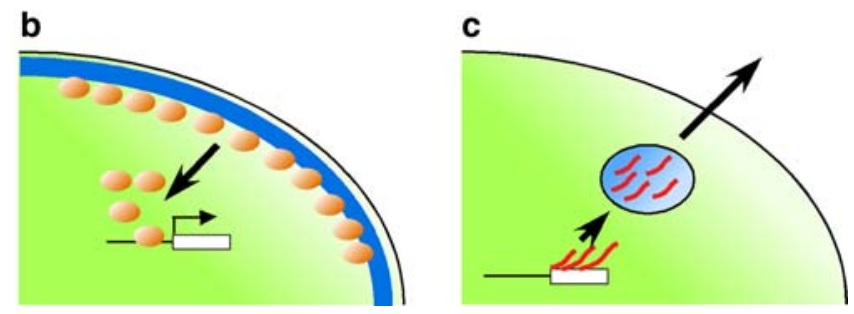

their availability at target genes. Sequestration can act both as a negative or a positive regulatory mechanism. Sequestration of an activator leads to repression, sequestration of a repression leads to activation. $\mathbf{c}$ Retention of partially or fully processed RNA within intranuclear compartments modulates RNA export rate

the nucleus. Several proteins are now known to accumulate, in particular nuclear locations in response to physiological cues and their sequestration is thought to contribute to their cellular function.

Prototypical example of sequestration as a regulatory mechanism is the accumulation of the ubiquitin ligases MDM2 and the von Hippel-Lindau tumor suppressor protein VHL in the nucleolus (Mekhail et al. 2005, 2007). These two proteins are responsible for the ubiquitination of the p53 tumor suppressor and the hypoxia-induced factor HIF, respectively. Modification of these two targets leads to their nuclear export and degradation in the cytoplasm. Since ubiquitination is a major regulatory mechanism in their function, the concentration of the ubiquitin ligase in the nucleus significantly determines their fate. Both MDM2 and VHL diffuse freely within the nucleus. However, in response to physiological cues, both MDM2 and VHL can become sequestered in the nucleolus, thus reducing their nucleoplasmic concentration and the extent of ubiquitination of their targets (Mekhail et al. 2005, 2007) (Fig. 1a). Sequestration of these proteins appears to occur by increased retention of diffusing molecules within the nucleolus. How this retention is regulated is currently unclear (Mekhail et al. 2005, 2007). The possibility that retention is a highly controlled mechanism comes from observations on nucleostemin, a nucleolar protein implicated in stem cell maintenance and cancer proliferation (Meng et al. 2007; Tsai and McKay 2005). The retention of nucleostemin in the nucleolus is controlled by a GTP-GDP binding cycle which might be directly or indirectly linked to signaling pathways. In addition to nucleostemin the localization of several additional GTP-binding domain containing nucleolar proteins seems to be controlled in a similar fashion (Meng et al. 2007; Tsai and McKay 2005).

Intranuclear sequestration can also be a means of negative regulation. The basic helix-loop-helix transcription factor Hand 1 is expressed in trophoblast stem cells and is required for their differentiation along several lineages (Martindill et al. 2007). Hand1 is sequestered in the nucleolus and upon stimulation during differentiation is rapidly released and moves into the nucleoplasm where it presumably acts on target genes. Hand 1 is retained in the nucleolus by its interaction with I-mfa, and this interaction is sensitive to phosphorylation of Hand1. Upon phosphorylation Hand1 dissociates from I-mfa and is released from the nucleolus. Interestingly, both the sequestration of Hand 1 in the nucleolus and its release are functionally important. Premature release of Hand1 triggers differentiation, whereas the presence of Hand1 is also required to maintain the stem-cell potential of trophoblast stem cells. Thus nucleolar sequestration of this key cell fate regulator serves a dual regulatory role (Martindill et al. 2007).

Most examples of intranuclear sequestration involve the nucleolus. However, the nuclear lamina has recently also emerged as a major site for transcription factor sequestration (Heessen and Fornerod 2007) (Fig. 1b). Prominent transcription factors which associate with the nuclear periphery either via interaction with the lamins or with the inner nuclear membrane proteins include c-fos, Oct-1 and $\mathrm{Rb}$ (Imai et al. 1997; Ivorra et al. 2006; Johnson et al. 2004). It is tempting to speculate that the association of these factors with the periphery reduces the intranuclear concentration and thus their availability at target genes (Heessen and Fornerod 2007). A physiological role for such peripheral sequestration is most strongly suggested by observations on the pro-proliferation factor c-fos which associates with the lamina in quiescent cells but is released upon entry of cells into the cell cycle correlating with activation of some of its target genes (Ivorra et al. 2006). Similarly, release of Oct-1 from the lamina correlates with activation of collagenase, one of its prime target genes (Imai et al. 1997). The sequestration of transcription factors at the nuclear periphery is a potentially powerful and simple mechanism of transcriptional regulation. It will be important to determine how widespread this mechanism is and how precisely the association of transcription factors with the lamina is controlled.

Dynamic trafficking of RNA is similarly used as a regulatory mechanism (Prasanth and Spector 2007). In particular, it appears that RNA retention can act as a quality 
control mechanism for RNA export. For one, incompletely spliced or processed RNAs are retained at their sites of transcription and not released into the nucleoplasm for export. In addition, pools of stable polyadenylated RNAs are enriched in the nuclear splicing factor compartments, although their function remains unclear (Huang et al. 1994). But retention is also emerging as a mechanism for regulation of specific RNAs. A non-coding RNA transcribed from the mouse Cat 2 gene encoding a cationic amino acid transporter is retained in the nucleus via a dedicated $3^{\prime}$ end containing extensive repeat sequences (Prasanth et al. 2005). These sequences are responsible for accumulation of the RNA in a nuclear compartment identified as paraspeckles (Fig. 1c). This retention serves a physiological function because upon stress the Cat2 RNA is cleaved, released from paraspeckles and rapidly exported into the cytoplasm for translation, thus ensuring a rapid physiological stress response. Although the Cat2 RNA is to-date the best characterized example of such retention, a similar mechanism is likely at work for the migration-stimulating factor (MSF) mRNA whose $3^{\prime}$ end resembles that of Cat 2 and is retained within the nucleus (Kay et al. 2005). Under conditions of MSF secretion the RNA appears to be released from the nucleus and rapidly translated. A further candidate for control via nuclear retention is the neuronspecific gomafu RNA which is retained in intranuclear compartments. Interestingly, gomafu does not seem to encode for any protein and might thus be a non-coding regulatory RNA which is retained in the nucleus (Sone et al. 2007).

\section{Conclusions}

The past few years have seen a dramatic change in how we view the cell nucleus. We have come to appreciate the presence of distinct structural elements within the nucleus, the presence of a multitude of intranuclear bodies and the fact that genomes are non-randomly organized within the nuclear space. One of the most consequential findings has been the realization that just about every aspect of nuclear organization is highly dynamic. Both proteins and RNAs move rapidly within the nucleus, and they only transiently interact with chromatin and nuclear bodies. It is now clear that the dynamic nature of nuclear components is a fundamental property and has implications for how molecules are targeted to their final destinations and for how intranuclear compartments form. Most importantly, it is becoming clear that the dynamic properties of nuclear proteins are critical for various mechanisms of physiological regulation, particularly via sequestration and retention of proteins and RNA. It is likely that these recently discovered examples of regulation by modulation of dynamic interactions within the cell nucleus are only the proverbial tip of the iceberg, and it stands to reason that we should consider the contribution of dynamic interactions and trafficking in any nuclear event we investigate in the future.

\section{References}

Andersen JS, Lam YW, Leung AK, Ong SE, Lyon CE, Lamond AI, Mann M (2005) Nucleolar proteome dynamics. Nature 433:77-83

Burke B, Stewart CL (2006) The laminopathies: the functional architecture of the nucleus and its contribution to disease. Annu Rev Genomics Hum Genet 7:369-405

Carrero G, Hendzel MJ, de Vries G (2006) Modelling the compartmentalization of splicing factors. J Theor Biol 239:298-312

Dundr M, Hoffmann-Rohrer U, Hu Q, Grummt I, Rothblum LI, Phair RD, Misteli T (2002) A kinetic framework for a mammalian RNA polymerase in vivo. Science 298:1623-1626

Dundr M, Hebert MD, Karpova TS, Stanek D, Xu H, Shpargel KB, Meier UT, Neugebauer KM, Matera AG, Misteli T (2004) In vivo kinetics of Cajal body components. J Cell Biol 164:831-842

Görisch SM, Lichter P, Rippe K (2005) Mobility of multi-subunit complexes in the nucleus: accessibility and dynamics of chromatin subcompartments. Histochem Cell Biol 123:217-228

Gorski SA, Dundr M, Misteli T (2006) The road much traveled: trafficking in the cell nucleus. Curr Opin Cell Biol 18:284-290

Gruenbaum Y, Margalit A, Goldman RD, Shumaker DK, Wilson KL (2005) The nuclear lamina comes of age. Nat Rev Mol Cell Biol 6:21-31

Handwerger KE, Gall JG (2006) Subnuclear organelles: new insights into form and function. Trends Cell Biol 16:19-26

Heessen S, Fornerod M (2007) The inner nuclear envelope as a transcription factor resting place. EMBO Rep 8:914-919

Hernandez-Verdun D (2006) The nucleolus: a model for the organization of nuclear functions. Histochem Cell Biol 126:135-148

Houtsmuller AB, Rademakers S, Nigg AL, Hoogstraten D, Hoeijmakers JH, Vermeulen W (1999) Action of DNA repair endonuclease ERCC1/XPF in living cells. Science 284:958-961

Huang S, Deerinck MH, Ellisman MH, Spector DL (1994) In vivo analysis of the stability and transport of nuclear poly(A)+ RNA. J Cell Biol 126:877-899

Imai S, Nishibayashi S, Takao K, Tomifuji M, Fujino T, Hasegawa M, Takano T (1997) Dissociation of Oct-1 from the nuclear peripheral structure induces the cellular aging-associated collagenase gene expression. Mol Biol Cell 8:2407-2419

Ivorra C, Kubicek M, Gonzalez JM, Sanz-Gonzalez SM, Alvarez-Barrientos A, O'Connor JE, Burke B, Andres V (2006) A mechanism of AP-1 suppression through interaction of c-Fos with lamin A/C. Genes Dev 20:307-320

Jiménez-García LF, Spector DL (1993) In vivo evidence that transcription and splicing are coordinated by a recruiting mechanism. Cell 73:47-59

Johnson BR, Nitta RT, Frock RL, Mounkes L, Barbie DA, Stewart CL, Harlow E, Kennedy BK (2004) A-type lamins regulate retinoblastoma protein function by promoting subnuclear localization and preventing proteasomal degradation. Proc Natl Acad Sci USA 101:9677-9682

Kay RA, Ellis IR, Jones SJ, Perrier S, Florence MM, Schor AM, Schor SL (2005) The expression of migration stimulating factor, a potent oncofetal cytokine, is uniquely controlled by $3^{\prime}$-untranslated region-dependent nuclear sequestration of its precursor messenger RNA. Cancer Res 65:10742-10749

Kruhlak MJ, Lever MA, Fischle W, Verdin E, Bazett-Jones DP, Hendzel MJ (2000) Reduced mobility of the alternate splicing factor 
(ASF) through the nucleoplasm and steady state speckle compartments. J Cell Biol 150:41-51

Lamond AI, Sleeman JE (2003) Nuclear substructure and dynamics. Curr Biol 13:R825-R828

Lanctot C, Cheutin T, Cremer M, Cavalli G, Cremer T (2007) Dynamic genome architecture in the nuclear space: regulation of gene expression in three dimensions. Nat Rev Genet 8:104-115

Martindill DM, Risebro CA, Smart N, Franco-Viseras Mdel M, Rosario CO, Swallow CJ, Dennis JW, Riley PR (2007) Nucleolar release of Hand 1 acts as a molecular switch to determine cell fate. Nat Cell Biol 9:1131-1141

McNally JG, Muller WG, Walker D, Wolford R, Hager GL (2000) The glucocorticoid receptor: rapid exchange with regulatory sites in living cells. Science 287:1262-1265

Meaburn KJ, Misteli T (2007) Cell biology: chromosome territories. Nature 445:379-781

Mekhail K, Khacho M, Carrigan A, Hache RR, Gunaratnam L, Lee S (2005) Regulation of ubiquitin ligase dynamics by the nucleolus. J Cell Biol 170:733-744

Mekhail K, Rivero-Lopez L, Al-Masri A, Brandon C, Khacho M, Lee $S$ (2007) Identification of a common subnuclear localization signal. Mol Biol Cell 18:3966-3977

Meng L, Zhu Q, Tsai RY (2007) Nucleolar trafficking of nucleostemin family proteins: common versus protein-specific mechanisms. Mol Cell Biol (in press)

Misteli T (2001) Protein dynamics: implications for nuclear architecture and gene expression. Science 291:843-847

Misteli T (2007) Beyond the sequence: cellular organization of genome function. Cell 128:787-800

Misteli T, Caceres JF, Spector DL (1997) The dynamics of a premRNA splicing factor in living cells. Nature 387:523-527

Phair RD, Misteli T (2000) High mobility of proteins in the mammalian cell nucleus. Nature 404:604-609
Phair RD, Scaffidi P, Elbi C, Vecerova J, Dey A, Ozato K, Brown DT, Hager G, Bustin M, Misteli T (2004) Global nature of dynamic protein-chromatin interactions in vivo: three-dimensional genome scanning and dynamic interaction networks of chromatin proteins. Mol Cell Biol 24:6393-6402

Prasanth KV, Spector DL (2007) Eukaryotic regulatory RNAs: an answer to the 'genome complexity' conundrum. Genes Dev 21:11-42

Prasanth KV, Prasanth SG, Xuan Z, Hearn S, Freier SM, Bennett CF, Zhang MQ, Spector DL (2005) Regulating gene expression through RNA nuclear retention. Cell 123:249-263

Ritland-Politz JC, Tufft RA, Prasanth KV, Baudendistel N, Fogarty KE, Lifshitz LM, Langowski J, Spector DL, Pederson T (2006) Rapid, diffusional shuttling of poly(A)-RNA between nuclear speckles and the nucleoplasm. Mol Cell Biol 17:1239-1249

Seksek O, Biwersi J, Verkman AS (1997) Translational diffusion of macromolecule-sized solutes in cytoplasm and nucleus. J Cell Biol 138:131-142

Shav-Tal Y, Darzacq X, Shenoy SM, Fusco D, Janicki SM, Spector DL, Singer RH (2004) Dynamics of single mRNPs in nuclei of living cells. Science 304:1797-1800

Sone M, Hayashi T, Tarui H, Agata K, Takeichi M, Nakagawa S (2007) The mRNA-like noncoding RNA Gomafu constitutes a novel nuclear domain in a subset of neurons. J Cell Sci 120:24982506

Spector DL (2003) The dynamics of chromosome organization and gene regulation. Annu Rev Biochem 72:573-608

Sprague BL, Muller F, Pego RL, Bungay PM, Stavreva DA, McNally JG (2006) Analysis of binding at a single spatially localized cluster of binding sites by fluorescence recovery after photobleaching. Biophys J 91:1169-1191

Tsai RY, McKay RD (2005) A multistep, GTP-driven mechanism controlling the dynamic coupling of nucleostemin. J Cell Biol 168:179-184 